\title{
Benefits and Limitations of Laparoscopic Myomectomy
}

\author{
Silwal Bal Mukunda, Yang Shen*, Shrestha Sugandha \\ Department of Obstetrics and Gynecology, Zhongda Hospital Affiliated to Southeast University, Nanjing, China \\ Email: *shenyang0924@sina.cn
}

How to cite this paper: Mukunda, S.B., Shen, Y. and Sugandha, S. (2019) Benefits and Limitations of Laparoscopic Myomectomy. Open Journal of Obstetrics and Gynecology, 9, 292-301. https://doi.org/10.4236/ojog.2019.93030

Received: February 10, 2019

Accepted: March 1, 2019

Published: March 4, 2019

Copyright $\odot 2019$ by author(s) and Scientific Research Publishing Inc. This work is licensed under the Creative Commons Attribution International License (CC BY 4.0).

http://creativecommons.org/licenses/by/4.0/ (c) (i) Open Access

\begin{abstract}
Uterine fibroids (also called leiomyoma or myoma) are the most common benign tumor among women of childbearing age. These are often discovered during bimanual examinations and/or ultrasound examinations. Laparoscopic myomectomy offers several benefits to patients such as less blood loss during operation, quick postoperative recovery and shorter hospital stay in comparison to open myomectomy. It has been widely used over the past decades as possible alternative to the traditional laparotomy. In spite of several benefits laparoscopic myomectomy has certain drawbacks such as prolonged time of anesthesia, time consuming procedure, comparatively expensive and weak strength of the uterine scar. Furthermore laparoscopic myomectomy is technically challenging procedure so it requires high degree of training and skill to perform it. Due to its several advantages, it should be taken into consideration for the removal of uterine fibroids.
\end{abstract}

\section{Keywords}

Laparoscopic Myomectomy, Recurrence, Uterine Rupture, Adhesion, Open Myomectomy, Uterine Fibroid

\section{Introduction}

Uterine fibroids (also called leiomyoma or myoma) are the most common benign tumor among women of childbearing age. Bimanual examinations and medical imaging are usually used to diagnose it [1]. Although Uterine fibroid is common, it can be asymptomatic in most of the women; however symptomatic patients can have a wide range of symptoms including dysfunctional uterine bleeding, mild to severe anemia, pressure effects on pelvic area, pelvic pain, infertility, and recurrent pregnancy loss [2]. At present, many minimally invasive approaches are available for the removal of myoma including open myomecto- 
my, vaginal myomectomy, laparoscopic myomectomy, single access laparoscopy, robotic assisted laparoscopy [3], uterine artery embolization, and uterine artery occlusion [4]. A laparoscopic myomectomy is a minimally invasive procedure that involves the surgical removal of uterine tumors known as fibroids by means of a laparoscope. Abdominal myomectomy used to be the first choice of surgical treatment for removal of uterine myoma in the past decades. However, with advancement in surgical approach and technique, minimally invasive technique such as laparoscopic myomectomy and many others has been developed alternative to open myomectomy [5]. This article mainly focuses on the benefits and disadvantages of Laparoscopic myomectomy on the basis of searched article. The aim of this paper is to present a comprehensive and up-to-date review on the topic of Laparoscopic Myomectomy.

\section{Methods}

Various Databases such as Pub Med, Web of Science, Cochrane were searched from 2010 to 2018 using the keywords such as Uterine fibroids, Laparoscopic Myomectomy alone and in combination of laparoscopic myomectomy with adhesions, uterine rupture, recurrence and technique. Additionally, above-mentioned database were also searched for the Abdominal Myomectomy. Only the relevant articles were included in this review article. The articles written in English language only were selected for this topic. The corresponding articles listed in the reference part were also meticulously scrutinized and searched for relevant literature. From above mentioned key words, around 6 articles relevant to our topic were found and included in my study (Figure 1).

\section{Basic Technique of Laparoscopic Myomectomy}

Depending on the preference of the surgeon, various techniques can be modified such as trochar placement to minimize bleeding during operation. A short overview of the basic steps of Laparoscopic Myomectomy is described below [6]. During the procedure, uterine manipulator is used which offers manipulation of uterus to create tension. Prior to incision of uterus for the removal of myoma, one vial of pitression (8-arginine vasopression) is injected along the planned serosal layer. Since it causes vascular spasm and uterine muscle contraction, it is effective in preventing the uterine bleeding during operations [6]. [7]. There are reported rare severe complications following the vasopression injection including pulmonary edema, severe hypotension and bradycardia with eventful cardiac arrest [6]. Therefore it is advocated that needle aspiration is mandatory prior to injection to avoid intravascular injection of this potent vasoconstrictor.

Following the vasopression injection, a monopolar instrument (hook or scissors) or a harmonic scalpel is used to make incision on serosal layer in order to decrease the risk of bleeding [8]. The length should accommodate the approximate diameter of the largest tumor. Once myoma pseudocapsule is reached, 


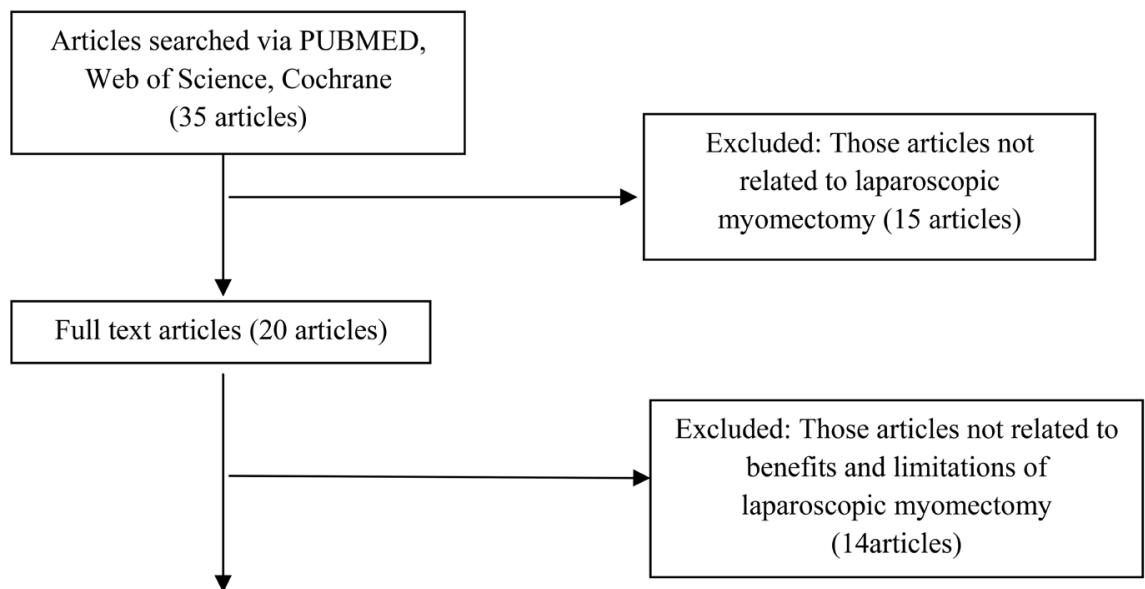

Final articles for review (6 articles)

Figure 1. Flowchart showing the article selection process included in the review.

leiomyoma screw or forceps are generally used to grasp the myoma,. Blunt probe or suction irrigator is used to dissect the pseudocapsule surrounding the leiomyoma that frees the tumor from adjacent myometrium. Myoma attached to the myometrium can be lysed using bipolar forceps or monopolar scissors. All the enucleated myoma are temporarily kept in the cul de sac and are taken out at the end of procedure using either electrical or mechanical morcellation. Prior to the closure of myometrium defect, single or multilayer closure should be taken into consideration to reduce the risk of hematoma, postoperative bleeding or uterine rupture in subsequent pregnancies [6] [8].

\section{Laparoscopic Myomectomy versus Open Myomectomy}

Jin et al. conducted meta-analysis in 2009 to determine the better method of myomectomy by comparing laparoscopic and open myomectomy for patients with fibroids. Randomized controlled trials included in this metaanalysis were performed between 1979 and 2007 on laparoscopic versus open myomectomy in which various operative parameters and outcomes were assessed [9]. According to his meta-analysis, laparoscopic myomectomy was associated with decreased blood loss during surgery. Data showed that postoperative pain was also diminished. However, it took longer operation time than abdominal myomectomy. Other parameters such as rate of recurrence of myoma and rate of postoperative pregnancy were similar in both LM and OM groups [9]. A recent Cochrane review (2014) proposed a meta-analysis in which nine randomized controlled trials were included (with a total of 808 women) to determine the benefits and disadvantages of laparoscopic myomectomy over open myomectomy. Postoperative pain (VAS-Visual Analog Scale was used to assess the degree of pain) and adverse events such as febrile morbidity, laparoconversion, intraoperative injury and drop in hemoglobin level were primary outcomes whereas duration of hos- 
pital stay, operative time and recurrence of uterine fibroids were secondary outcomes [10]. According to Cochrane review, following parameters such as postoperative pain, febrile morbidity and duration of hospital stay were compared between laparoscopic myomectomy and open myomectomy group. The authors concluded that LM group had less postoperative pain, reduced febrile morbidity and shorter hospital stay than OM group [10]. Similar benefits of laparoscopic myomectomy were observed in a prospective, non-randomized study conducted by Kalogiannidis et al. [11]. Chen et al. analyzed surgical outcomes of both LM and OM procedures from eight hospitals in Vancouver Coastal Health and Providence Health Region from British Columbia. The authors found that although OM is technically easier than LM, LM leads to faster and easier recovery [12].

\section{Considerations and Concerns about Laparoscopic Myomectomy}

\subsection{Postoperative Adhesions}

Risk of pelvic adhesions is associated with most of surgical intervention including both laparoscopic and laparotomic approach [4] [15]. Adhesions refer to fibrous bands between two anatomical sites often as a result of injury during surgery which are associated with important morbidity of patients because it can lead to small bowel obstruction, chronic pelvic pain, infertility and laborious subsequent surgery [16] [17]. Myoma located posteriorly was found to be more likely associated with higher risk of adhesions than the myoma located on anterior side of uterus [15] [18]. There are various important factors which can influence the development of adhesions including number and size of myoma [19]. Excessive accumulation of suturing materials is also factor that increase the risk of adhesions formation [15] [20]. Adhesions are known to be an important burden to patients therefore several principles must be taken into consideration to minimize the risk of adhesion development such as using a best anti-adhesion agent, minimizing cautery time and using less sutures [4] [15]. Adhesions impair the interaction between the oviduct and the ovary. Adhesions are assumed to cause $20 \%-30 \%$ of female infertility [21] [22]. Keeping this in mind, surgeon should pay attention to reduce adhesion formation during laparoscopic myomectomy. The pressure used during laparoscopic myomectomy to maintain pneumoperitoneum results in tissue hypoxia. As a result of this, fibrinolytic system is disturbed which is considered as important factor in adhesion formation [23]. In a prospective blinded observational study Tinelli et al. examined the effect of an anti-adhesion agent used during laparoscopic and abdominal myomectomy. A total of 546 patients with similar baseline characteristics and almost similar size of the fibroid were examined in the subsequent procedure for various reasons. The incidence of adhesions in the different groups was as follows: abdominal myomectomy (AM) without adhesion barrier (AB; 28.1\%), laparoscopic myomectomy (LM) without adhesion barrier (22.6\%), AM with adhesion 
barrier (22\%), and LM with adhesion barrier (15.9\%) [24]. Kubinova et al assessed adhesions during a second-look laparoscopy for adhesiolysis after abdominal or laparoscopic myomectomy. Their study found $96.65 \%$ of patients developed adhesions following laparotomy $(n=28)$ compared with $71.43 \%$ of patients following laparoscopy $(n=68)$ [25]. Some of the studies have discovered that the occurrence of adhesions is minimized following laparoscopic technique. However, the use of laparoscopy is unable to prevent adhesion formation completely [4]. In addition to this, skill of surgeon may also have an impact on the development of adhesions. The simple principle like gentle tissue handling, constant tissue moistening and reduced use of electrocautery may decrease the development of adhesions [19]. Finally, surgeons should consider the use of an anti-adhesion agent before the closure of abdomen in order to minimize the formation of adhesions. Comparison between laparoscopic myomectomy and open laparotomy was performed in which several benefits were seen among laparoscopic group such as less postoperative pain, less blood loss during surgery and shorter hospital (Table 1).

\subsection{Uterine Rupture}

The chief concern following laparoscopic myomectomy in women of reproductive age is the risk of uterine rupture during subsequent pregnancy [26]. Although it is quite rare event, some study revealed that incidence of uterus rupture following laparoscopic myomectomy is $0.7 \%-1 \%$. This is because various factors might contribute to the development of a weak scar with the subsequent risk for uterine rupture [27] [28]. The overuse of electrocoagulation to stop bleeding can result in tissue necrosis which often complicate wound healing process [29]. There are other several factors which could influence wound healing process in the myometrium such as presence of infection or hematoma formation within myometrium and extent of local tissue destruction [30]. Another possible influencing factor for the development of weak scar may be an inadequate suturing of the myometrial defect. In order to eliminate dead space and to achieve complete wound closure, multilayer closure of myometrium is necessary [31]. Kumakiri et al. discussed feasibility and safety of vaginal birth after laparoscopic myomectomy by using protocols for a vaginal birth following laparoscopic myomectomy. The authors concluded that uterine rupture during pregnancy following LM is rare and vaginal birth after LM appears to be safe in selected patients who met their criteria. Therefore, if a expert surgeon operate LM, possibility of uterine rupture is rare [31]. Bujold et al. and his team conducted the impact of single-layer closure or double-layer closure on uterine rupture in which they found that single-layer closure was associated with higher risk of dehiscence when compared with two-layer technique [32]. Depending on depth of the myometrium defect after laparoscopic myomectomy, the multilayer suture should be taken into consideration to reduce dead space and assure a good wound healing [4] [33]. The uterine rupture mostly occurs between 17 and 40 weeks of gestation [30]. The majority takes place before the start of labor or in 
Table 1. Primary and secondary outcomes for laparoscopic and open myomectomy reported in different studies and meta-analysis.

\begin{tabular}{lll}
\hline LM VS OM & Seracchioli et al. 2000 [13] & Holzer et al. 2006 [14] \\
\hline Total no. of patients & $131(66$ versus 65$)$ & $40(19$ versus 21) \\
Age (years) & $34.00 \pm 4.11$ vs $33.97 \pm 4.79$ & $40(31-45)$ vs $40(28-49)$ \\
Total no. of fibroids & $2.94 \pm 1.53$ vs $2.75 \pm 1.98$ & $2(1-4)$ vs $3(1-7)$ \\
Diameter of the largest fibroids $(\mathrm{cm})$ & $7.07 \pm 2.54$ vs $7.47 \pm 2.60$ & $7(4-10)$ vs $5(3-11)$ \\
Length of surgery $(\mathrm{min})$ & $100.23 \pm 38.34$ vs $88.85 \pm 26.91$ & $99 \pm 37$ vs $68 \pm 22$ \\
Hemoglobin drop $(\mathrm{gm} / \mathrm{dl})$ & $1.33 \pm 1.23$ vs $2.17 \pm 1.57$ & \\
Complications & Fever: $1 / 20$ vs $1 / 20$ & None \\
Length of hospital stay & $75.61 \pm 37.09$ vs $142.80 \pm 34.60$ & $\begin{array}{l}\text { None } \\
2.28 \pm 1.38 \text { vs } 4.30 \pm 1.63\end{array}$ \\
$\begin{array}{l}\text { Postoperative pain } \\
\text { assessed by visual analog scale }\end{array}$ & None & $\begin{array}{l}\text { Mean overall VAS-score } \\
\text { at } 24 \text { h, } 48 \mathrm{~h}, 72 \mathrm{~h} \\
\text { postoperatively }\end{array}$ \\
\hline
\end{tabular}

the early middle third trimester therefore patients as well as patients party should be well counseled about the possibility of uterine rupture by the doctors [4].

\subsection{Myoma Recurrence}

Age of patients, preoperative number of uterine fibroids, preoperative size of uterine fibroids, presence of associated pelvic disease and child birth after surgery are associated with the recurrence of uterine fibroids [34] [35]. These days, most of skilled surgeon choose laparoscopic myomectomy to remove myoma as it is safe procedure for the patients and comparatively less invasive than abdominal approach. There is still debate going on among authors regarding the risk of myoma recurrence following LM because surgeons often find difficult to palpate the small intramural myomas and leave it during laparoscopic approach. These unremoved small myomas during LM could grow and could be responsible for the recurrence of symptoms in the future. So when numerous small fibroids are found during preoperative work-up, laparoscopic is not the most suitable approach for dealing with them. Dordot et al. concluded that the cumulative recurrence risk was $12.7 \%$ at 2 years and $16.7 \%$ at 5 years. They found that number of myomas and nulliparity was linked with the recurrence of myoma following laparoscopic myomectomy [36].

\subsection{Fertility and Myomectomy}

Another area of interest is fertility outcomes following laparoscopic myomectomy. $10 \%$ of women with infertility is estimated to have uterine fibroids. Myomas particularly submucosal are associated with reduced fertility and increased miscarriage rate. Some studies have found that pregnancy rates were improved in women who received treatment for the distortion created by submucosal fibroids [37]. Women with subserosal fibroids have no association with infertility and 
miscarriage risk. Seracchioli et al. enrolled 131 patients with otherwise unexplained infertility and found no significant difference in the pregnancy and abortion rate between the Laparoscopic and laparotomic group [11] [38].

\section{Discussion}

Despite of being more popular among surgeon for the removal of myoma, it has several disadvantages which may affect the health of patients. Several articles have discussed the potential disadvantages of LM such as postoperative adhesion, risk of uterine rupture and recurrence of uterine fibroids following LM. As discussed earlier, preoperative number particularly 2 or more fibroids have strong link with the recurrence of fibroids following LM. Several other articles have found prescence of multiple myomas contributed to recurrence of fibroids [34] [35]. The removal of all fibroids through this procedure is quite tough so unremoved myoma could grow later and casuse pain and discomfort on the pelvic region. Therefore when preoperative work-up finds multiple fibroids, surgeon should think of laparotomy to prevent recurrence of fibroids. The another concern of LM for women of childbearing age is uterine rupture during pregnancy. Some articles discussed several risk factors for the risk of uterine rupture following LM such as inadequate suturing of myometrial defect and impaired wound healing due to overuse of eletrocoagulation [4] [39]. So in order to minimize the risk of uterine rupture, surgeon must judiciously use cautry to stop bleeding and suture myometrial tightly to eliminate dead space. However Hurst et al. found that uterine rupture is quite rare event after laparoscopy [8]. There is risk of postoperative adhesion formation after every surgical procedure which causes several unwanted problems to the patients such as small bowel obstruction, infertility, chronic pelvic pain. The pressure used during laparoscopic procedure to maintain pneumoperitonium play key role in the adhesion formation [23]. Nevertheless, adhesion formation is relatively quite low after laparoscopy than laparotomy [4]. The potential disadvantages of laparoscopic myomectomy are technically demanding procedure which requires a high degree of training and skill. In spite of several disadvantages, surgeon often uses laparoscopy for the removal of fibroids because it possess more benefits than disadvantages. Several studies have discussed the benefits of laparoscopic approach. sertoli holzer (refreneces) The benefits include shorter hospital stay, less postoperative pain, quick recovery, less blood loss during surgery if performed by well skilled surgeon. Another advantage of laparoscopy is less adhesion formation following laparoscopy than laparotomy.

\section{Conclusion}

Laparoscopic myomectomy, a popular minimal invasive technique, has been widely used for removal of uterine fibroid over the past decades. The main drawbacks of LM are high chance of uterine rupture, high risk of recurrence and technically demanding procedure which require a high degree of training and skill. One of the great concerns regarding the laparoscopic myomectomy is its 
higher cost than open laparoscopic myomectomy. Initially it seems costly procedure due to its higher direct costs but shorter hospital stay, quick postoperative recovery and lower analgesic requirement might compensate the higher direct costs. Moreover, laparoscopy minimizes the risk of adhesion formation. Therefore laparoscopy should be standard approach for myomectomy. Lastly, further prospective studies have to be conducted to compare long-term outcome Laparoscopic myomectomy including skill evaluation.

\section{Acknowledgements}

We would like to thank the faculty members of Department of Obstetrics and Gynecology, Zhongda Hospital affiliated to Southeast University for their coordination.

\section{Conflicts of Interest}

The authors declare no conflicts of interest regarding the publication of this paper.

\section{References}

[1] Cheng, M.-H. and Wang, P.-H. (2008) Uterine Myoma: A Condition Amendable to Medical Therapy? Expert Opinion on Emerging Drugs, 13, 119-133. https://doi.org/10.1517/14728214.13.1.119

[2] Stewart, E.A. (2001) Uterine Fibroids. The Lancet, 357, 293-298. https://doi.org/10.1016/S0140-6736(00)03622-9

[3] Magrina, J. (2007) Robotic Surgery in Gynecology. European Journal of Gynaecological Oncology, 28, 77-82.

[4] Herrmann, A. and De Wilde, R.L. (2014) Laparoscopic Myomectomy-The Gold Standard. Gynecology and Minimally Invasive Therapy, 3, 31-38. https://doi.org/10.1016/j.gmit.2014.02.001

[5] Parker, W.H. (2007) Uterine Myomas: Management. Fertility and Sterility, 88, 255-271. https://doi.org/10.1016/j.fertnstert.2007.06.044

[6] Mattei, A., et al. (2011) Techniques of Laparoscopic Myomectomy. Reproductive Biomedicine Online, 23, 34-39. https://doi.org/10.1016/j.rbmo.2010.09.011

[7] Shimanuki, H., et al. (2006) The Effect of Vasopressin on Local and General Circulation during Laparoscopic Surgery. Journal of Minimally Invasive Gynecology, 13, 190-194. https://doi.org/10.1016/j.jmig.2006.01.015

[8] Hurst, B.S., Matthews, M.L. and Marshburn, P.B. (2005) Laparoscopic Myomectomy for Symptomatic Uterine Myomas. Fertility and Sterility, 83, 1-23. https://doi.org/10.1016/j.fertnstert.2004.09.011

[9] Jin, C., et al. (2009) Laparoscopic versus Open Myomectomy-A Meta-Analysis of Randomized Controlled Trials. European Journal of Obstetrics \& Gynecology and Reproductive Biology, 145, 14-21. https://doi.org/10.1016/j.ejogrb.2009.03.009

[10] Chittawar, P.B., et al. (2014) Minimally Invasive Surgical Techniques versus Open Myomectomy for Uterine Fibroids. Cochrane Database of Systematic Reviews, No. 10, CD004638. https://doi.org/10.1002/14651858.CD004638.pub3

[11] Kalogiannidis, I., et al. (2010) Laparoscopically Assisted Myomectomy versus Abdominal Myomectomy in Short-Term Outcomes: A Prospective Study. Archives of 
Gynecology and Obstetrics, 281, 865-870.

https://doi.org/10.1007/s00404-009-1187-9

[12] Chen, I., et al. (2014) Laparoscopic versus Abdominal Myomectomy: Practice Patterns and Health Care Use in British Columbia. Journal of Obstetrics and Gynaecology Canada, 36, 817-821. https://doi.org/10.1016/S1701-2163(15)30484-9

[13] Seracchioli, R., et al. (2000) Fertility and Obstetric Outcome after Laparoscopic Myomectomy of Large Myomata: A Randomized Comparison with Abdominal Myomectomy. Human Reproduction, 15, 2663-2668. https://doi.org/10.1093/humrep/15.12.2663

[14] Holzer, A., et al. (2006) Laparoscopic versus Open Myomectomy: A Double-Blind Study to Evaluate Postoperative Pain. Anesthesia \& Analgesia, 102, 1480-1484. https://doi.org/10.1213/01.ane.0000204321.85599.0d

[15] Ahmad, G., et al. (2008) Barrier Agents for Adhesion Prevention after Gynaecological Surgery. Cochrane Database of Systematic Reviews, No. 2, CD000475.

[16] Cezar, C., et al. (2016) How to Avoid Risks for Patients in Minimal-Access Trials: Avoiding Complications in Clinical First-in-Human Studies by Example of the ADBEE Study. Best Practice \& Research Clinical Obstetrics \& Gynaecology, 35, 84-96. https://doi.org/10.1016/j.bpobgyn.2015.11.004

[17] Diamond, M.P. and Freeman, M.L. (2001) Clinical Implications of Postsurgical Adhesions. Human Reproduction Update, 7, 567-576.

https://doi.org/10.1093/humupd/7.6.567

[18] Ellis, H. and Crowe, A. (2009) Medico-Legal Consequences of Post-Operative Intra-Abdominal Adhesions. International Journal of Surgery, 7, 187-191. https://doi.org/10.1016/j.ijsu.2009.04.004

[19] Takeuchi, H., et al. (2008) Influencing Factors of Adhesion Development and the Efficacy of Adhesion-Preventing Agents in Patients Undergoing Laparoscopic Myomectomy as Evaluated by a Second-Look Laparoscopy. Fertility and Sterility, 89, 1247-1253. https://doi.org/10.1016/j.fertnstert.2007.05.021

[20] Bechev, B., et al. (2014) “Second Look” after Laparoscopic Myomectomy-Is That a Prevention of Postoperative Adhesions. Akusherstvo i Ginekologiia, 53, 18-21.

[21] Trew, G. (2006) Postoperative Adhesions and Their Prevention. Reviews in Gynaecological and Perinatal Practice, 6, 47-56. https://doi.org/10.1016/j.rigapp.2006.02.001

[22] DeWilde, R.L. and Trew, G. (2007) Postoperative Abdominal Adhesions and Their Prevention in Gynaecological Surgery. Expert Consensus Position. Gynecological Surgery, 4, 161-168. https://doi.org/10.1007/s10397-007-0338-x

[23] Ott, D.E. (2008) Laparoscopy and Adhesion Formation, Adhesions and Laparoscopy. Seminars in Reproductive Medicine, 26, 322-330.

https://doi.org/10.1055/s-0028-1082390

[24] Tinelli, A., et al. (2011) Adhesion Formation after Intracapsular Myomectomy with or without Adhesion Barrier. Fertility and Sterility, 95, 1780-1785. https://doi.org/10.1016/j.fertnstert.2010.12.049

[25] Kubinova, K., et al. (2012) Reproduction after Myomectomy: Comparison of Patients with and without Second-Look Laparoscopy. Minimally Invasive Therapy \& Allied Technologies, 21, 118-124. https://doi.org/10.3109/13645706.2011.573797

[26] Levi, A.A. (1961) Rupture of the Pregnant Uterus: Relationship to Previous Myomectomy. Obstetrics \& Gynecology, 18, 223-229.

[27] Garnet, J.D. (1964) Uterine Rupture during Pregnancy: An Analysis of 133 Patients. 
Obstetrics \& Gynecology, 23, 898-905.

[28] Golan, D., et al. (1990) Early Spontaneous Rupture of the Post Myomectomy Gravid Uterus. International Journal of Gynecology \& Obstetrics, 31, 167-170. https://doi.org/10.1016/0020-7292(90)90716-X

[29] Dubuisson, J.-B., et al. (1998) Second Look after Laparoscopic Myomectomy. Human Reproduction (Oxford, England), 13, 2102-2106. https://doi.org/10.1093/humrep/13.8.2102

[30] Parker, W.H., et al. (2010) Risk Factors for Uterine Rupture after Laparoscopic Myomectomy. Journal of Minimally Invasive Gynecology, 17, 551-554. https://doi.org/10.1016/j.jmig.2010.04.015

[31] Kumakiri, J., et al. (2008) Prospective Evaluation for the Feasibility and Safety of Vaginal Birth after Laparoscopic Myomectomy. Journal of Minimally Invasive Gynecology, 15, 420-424. https://doi.org/10.1016/j.jmig.2008.04.008

[32] Bujold, E., et al. (2002) The Impact of a Single-Layer or Double-Layer Closure on Uterine Rupture. American Journal of Obstetrics and Gynecology, 186, 1326-1330. https://doi.org/10.1067/mob.2002.122416

[33] Pistofidis, G., et al. (2012) Report of 7 Uterine Rupture Cases after Laparoscopic Myomectomy: Update of the Literature. Journal of Minimally Invasive Gynecology, 19, 762-767. https://doi.org/10.1016/j.jmig.2012.07.003

[34] Shiota, M., et al. (2012) Recurrence of Uterine Myoma after Laparoscopic Myomectomy: What Are the Risk Factors? Gynecology and Minimally Invasive Therapy, 1, 34-36. https://doi.org/10.1016/j.gmit.2012.08.003

[35] Yoo, E.-H., et al. (2007) Predictors of Leiomyoma Recurrence after Laparoscopic Myomectomy. Journal of Minimally Invasive Gynecology, 14, 690-697. https://doi.org/10.1016/j.jmig.2007.06.003

[36] Doridot, V., et al. (2001) Recurrence of Leiomyomata after Laparoscopic Myomectomy. The Journal of the American Association of Gynecologic Laparoscopists, 8, 495-500. https://doi.org/10.1016/S1074-3804(05)60610-X

[37] Obstetricians, A.C.o. and Gynecologists, ACOG Practice Bulletin (2008) Alternatives to Hysterectomy in the Management of Leiomyomas. Obstetrics and Gynecology, 112, 387.

[38] Palomba, S., et al. (2007) A Multicenter Randomized, Controlled Study Comparing Laparoscopic versus Minilaparotomic Myomectomy: Short-Term Outcomes. Fertility and Sterility, 88, 942-951. https://doi.org/10.1016/j.fertnstert.2006.12.048

[39] Dubuisson, J.-B., et al. (2000) Pregnancy Outcome and Deliveries Following Laparoscopic Myomectomy. Human Reproduction, 15, 869-873.

https://doi.org/10.1093/humrep/15.4.869

\section{Abbreviations}

LM: Laparoscopic Myomectomy

OM: Open Myomectomy 